\title{
ACKNOWLEDGMENT TO REFEREES
}

\section{Reviewers 2015}

(C) Springer-Verlag Italia 2016

The Editors and Springer gratefully acknowledge the work and expertise provided by all who have kindly reviewed for the journal from January 2015 to December 2015. Without their experience and invaluable assistance, we would not have been able to expeditiously review all submitted papers.

Adolfsson L. (Sweden)

Aik S. (Malaysia)

Begue T. C. (France)

Birkholtz F. F. (South Africa)

Blokhuis T. J. (Netherlands)

Blonna D. (Italy)

Bose D. (United Kingdom)

Burkhart K. (Germany)

Calder P. (United Kingdom)

den Hartog D. (Netherlands)

Donnan L. T. (Australia)

Eilertsen L. (Norway)

Ekholm C. (Sweden)

Eygendaal D. (Netherlands)

Faymonville C. (Germany)

Fernandez F. (Germany)

Ferreira N. (South Africa)

Giotakis N. (United Kingdom)
Harwood P. (United Kingdom)

Hosny G. (Egypt)

Jaeger M. (Germany)

James L. (United Kingdom)

Lambert S. (United Kingdom)

Langendörfer M. (Germany)

Lavini F. (Italy)

Lenich A. (Germany)

Mittlmeier T. (Germany)

Monsell F. (United Kingdom)

Mueller M. (Germany)

Narayan B. (United Kingdom)

Oleksak M. (Bermuda)

Penn-Barwell J. (United Kingdom)

Ploegmakers J. (Netherlands)

Qureshi A. (United Kingdom)

Reigstad O. (Norway)

Robinson P. (Australia)
Rutherford Davies J. (United Kingdom) Seybold D. (Germany) Shariff R. (United Kingdom)

Sharma H. (United Kingdom)

Sletten I. (Norway)

Sprott D. (United Kingdom)

Taylor M. (United Kingdom)

Trompeter A. (United Kingdom)

van der Zwan A. (Netherlands)

van Riet R. (Belgium)

von Laer L. (Switzerland)

Walton R. (United Kingdom)

W-Dahl A. (Schweden)

Werner B. (Germany)

Young J. (United Kingdom)

Youngman J. (United Kingdom) 